\title{
Winter Wheat Yield Prediction Using Convolutional Neural Networks from Environmental and Phenological Data
}

\section{Amit Kumar Srivastava}

University of Bonn

Nima Safaei ( $\square$ nima-safaei@uiowa.edu )

University of lowa

Saeed Khaki

lowa State University

Gina Lopez

University of Bonn

Wenzhi Zeng

Wuhan University

Frank Ewert

University of Bonn

Thomas Gaiser

University of Bonn

Jaber Rahimi

Karlsruhe Institute of Technology (KIT), Atmospheric Environmental Research (IMK-IFU)

\section{Research Article}

Keywords: convolutional neural network (CNN), crop genotype, weather, soil, management practices, machine learning $(\mathrm{ML})$

Posted Date: August 11th, 2021

DOl: https://doi.org/10.21203/rs.3.rs-789462/v1

License: (c) (i) This work is licensed under a Creative Commons Attribution 4.0 International License. Read Full License 


\title{
Winter Wheat Yield Prediction Using Convolutional Neural Networks from Environmental and Phenological Data
}

\author{
Amit Kumar Srivastava ${ }^{1}$, Nima Safaei ${ }^{2,+, *}$, Saeed Khaki ${ }^{3,+, *}$, Gina Lopez ${ }^{1}$, Wenzhi Zeng ${ }^{4}$, \\ Frank Ewert $^{1}$, Thomas Gaiser ${ }^{1}$, and Jaber Rahimi ${ }^{5}$ \\ ${ }^{1}$ Institute of Crop Science and Resource Conservation, University of Bonn, Bonn, 53111, Germany \\ ${ }^{2}$ Department of Business Analytics, Tippie College of Business, University of lowa, lowa, United States \\ ${ }^{3}$ Industrial and Manufacturing Systems Engineering Department, lowa State University, Ames, United States \\ ${ }^{4}$ State Key Laboratory of Water Resources and Hydropower Engineering Science, Wuhan University, Wuhan, \\ 430072, China \\ ${ }^{5}$ Karlsruhe Institute of Technology (KIT), Institute of Meteorology and Climate Research, Atmospheric \\ Environmental Research (IMK-IFU), Karlsruhe, Germany \\ *nima-safaei@uiowa.edu, skhaki@iastate.edu \\ +these authors contributed equally to this work
}

\begin{abstract}
Crop yield forecasting depends on many interactive factors including crop genotype, weather, soil, and management practices. This study analyzes the performance of machine learning and deep learning methods for winter wheat yield prediction using extensive datasets of weather, soil, and crop phenology. We propose a convolutional neural network (CNN) which uses the 1-dimentional convolution operation to capture the time dependencies of environmental variables. The proposed CNN, evaluated along with other machine learning models for winter wheat yield prediction in Germany, outperformed all other models tested. To address the seasonality, weekly features were used that explicitly take soil moisture and meteorological events into account. Our results indicated that nonlinear models such as deep learning models and XGboost are more effective in finding the functional relationship between the crop yield and input data compared to linear models and deep neural networks had a higher prediction accuracy than XGboost. One of the main limitations of machine learning models is their black box property. Therefore, we moved beyond prediction and performed feature selection, as it provides key results towards explaining yield prediction (variable importance by time). As such, our study indicates which variables have the most significant effect on winter wheat yield.
\end{abstract}

\section{Introduction}

The threat to food security from climate change is a critical issue given the world population is expected to reach 9 Billion by 2050 . Governments across the globe need to be well-equipped to deal with supply shocks in major cereals ${ }^{1}$. Moreover, farmers and growers rely mostly on past experiences and historical time-series data of the crop yields and weather events to make important decisions about agro-management practices in the current year. Crop yield prediction is one of the challenging problems in precision agriculture as the yields depend on several factors such as climate, weather, soil, and crop management ${ }^{2}$. In this regard, machine learning (ML) is a practical approach that can provide yield predictions and has emerged as promising technology over the past years that can potentially aid farmers' decision making ${ }^{3,4}$ and informed actions in real-world scenarios without or with minimal human interventions ${ }^{5-8}$. One of the main advantages of ML approaches is that they are capable of autonomously solving large nonlinear problems using datasets compiled from different sources. It provides a flexible and yet powerful framework for not only data-driven decision-making but also for the incorporation of expert knowledge into the system $^{9,10}$. Additionally, the (ML) approaches have emerged as promising alternative and complementary tools to the crop models which are constrained by several challenges including the availability of data to parameterize, calibrate and validate the models before their application ${ }^{11}$. However, the lack of spatial and temporal data that covers a range of production and management inputs (e.g. planting date, fertilizer application rate) to efficiently train the ML models is a limitation that needs to be overcome ${ }^{12}$. Several machine learning models such as CNN, SVM, OLS ${ }^{12-17}$ are being used in crop detection and yield forecasting. Though high prediction accuracy is the main consideration when using artificial intelligence models, good stability and less computational workload should also be considered ${ }^{18}$. Some models are inherently unstable and may produce less 
accurate estimates when forecasting with new data sets. Thus, the aims of this study are to (1) evaluate the accuracy and interpretability of ML models for forecasting winter wheat yield; (2) evaluate the effects of different climate variables on crop yield; and (3) establish and evaluate the applicability of the general model in a temperate climate.

\section{Methods}

\section{DATA ACQUISITION}

The data analyzed in the current study included yield performance, management, weather, and soil information for the entire territory of Germany.

\section{Weather data}

Minimum and maximum temperature, radiation, precipitation, relative humidity, and wind speed data at a daily time scale were obtained from the Deutscher Wetterdienst (DWD) for 41 years (ranging from 1979-2019) and interpolated to a $1 \mathrm{~km}$ grid as described in Zhao et al. (2015) ${ }^{19}$ and aggregated to weekly values at NUTS3 (Nomenclature of Territorial Units for Statistics $^{20}$ ) level for input to the ML model. For aggregation to NUTS3 level, weights averages were computed using weights corresponding to the fraction of each grid cell that was under agricultural land-use which were assigned based on CORINE Land Cover 2006 land-use data at an original resolution of $250 \mathrm{~m}^{21}$.

\section{Soil data}

For input to the ML model, soils were aggregated to the DWD grids by selecting the majority soil type, considering only soils corresponding to the same agricultural land-use categories from CORINE 2006 used for the climate. The soil data source is the soil reconnaissance map of Germany available at 1:1,000,000 differentiated by land-use commonly known as BÜK1000N (BGR). The soil-related parameters used were soil texture values (e.g. percentage of sand, silt, and clay), crop available water at field capacity, saturation and the wilting point, and bulk density available up to soil depth of 1.3 meters. The spatial resolution of the soil data was $1 \mathrm{~km}^{2}$.

\section{Crop yield statistics}

Crop yield statistics of about 271 counties at the subnational NUTS $3{ }^{22}$ level for winter wheat across Germany were analyzed for years 1999 to 2019. The NUTS3 level of Germany equals county ("Kreise"). NUTS3 crop yields were extracted from the regional database of Germany ${ }^{23}$.

\section{Crop phenology data}

Observations of winter wheat sowing, anthesis, and harvest were accessed from the DWD phenology database ${ }^{24}$. Data at the NUTS3 level were resampled to DWD $1 \mathrm{~km}$ simulation grids by joining grids to NUTS3 based on their centroid. In the next step, we randomly sampled 50 weather and soil from each county and took the average of these samples to get representative values for both weather and soil (Figure 1) data did not have any missing values, but we found the daily data to be more granular than necessary to reveal the essential information. As a result, we took the weekly average and achieved a 365:52 ratio of dimension reduction. Such preprocessing of the weather data substantially reduced the number of parameters of machine learning methods. Table 1 shows the summary statistics of winter wheat data. There are different sowing/harvest dates for different counties. This results in different growing season lengths. The histogram of growing season length across all counties in 2019 is shown in Figure 2.

\begin{tabular}{ll}
\hline Summary Statistics & Winter Wheat Data \\
\hline Total number of locations & 271 \\
Year range & $1999-2019$ \\
Mean Yield & 6.3 \\
Standard deviation of yield & 0.98 \\
Minimum yield & 2.2 \\
Maximum yield & 9.5 \\
Number of weather components & 6 \\
Number of soil components & 7 \\
Number of phenology components & 3 \\
Number of observations & 5692 \\
\hline
\end{tabular}

Table 1. Summary statistics of the data used in the study. The unit of yield is tons $\mathrm{ha}^{-1}$. 


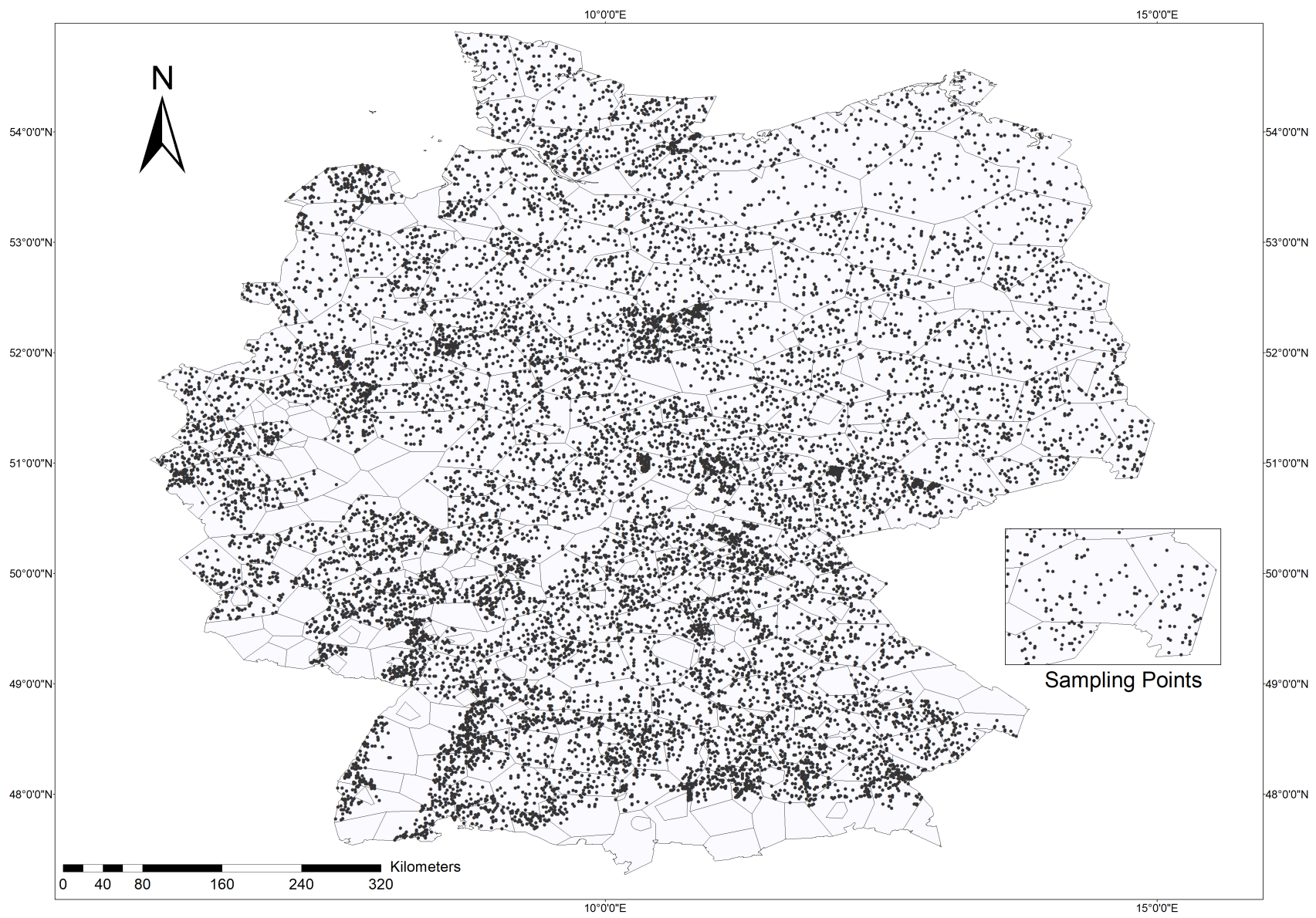

Figure 1. Sampling points for the weather and soil data collection in 271 counties across Germany.

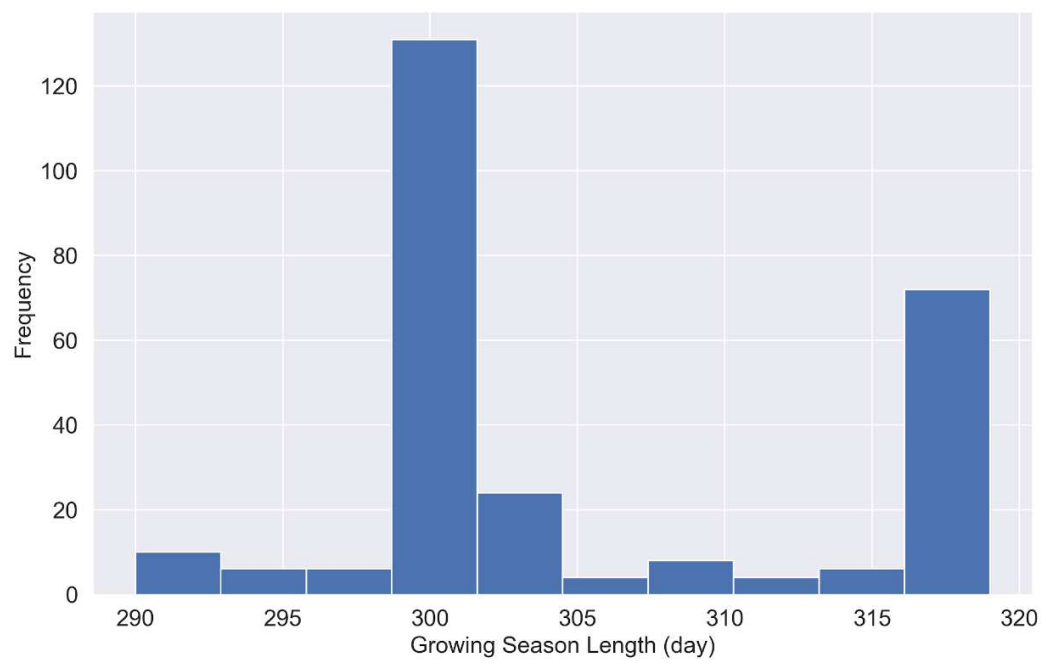

Figure 2. Frequency distribution of growing season length (from sowing to harvest) across the 271 counties in the year 2019. 


\section{ML models description \\ Proposed method (CNN)}

The proposed method combines convolutional neural networks and fully-connected (FC) neural networks for crop yield prediction. The convolutional neural network part of the model takes in the weather variables measured through growing season as input and captures their temporal and nonlinear effects using 1-dimentional convolution operations. All six weather variables go into the same CNN model separately, and we concatenate their corresponding output of the CNN. The soil and phenology data go to a fully-connected neural network with two layers. We combine the high-level features of the CNN with the output of the fully-connected neural network for soil and phenology data. Finally, the combined features go to three other FC layers before final yield prediction. The modeling architecture of the model is shown in Figure 3.

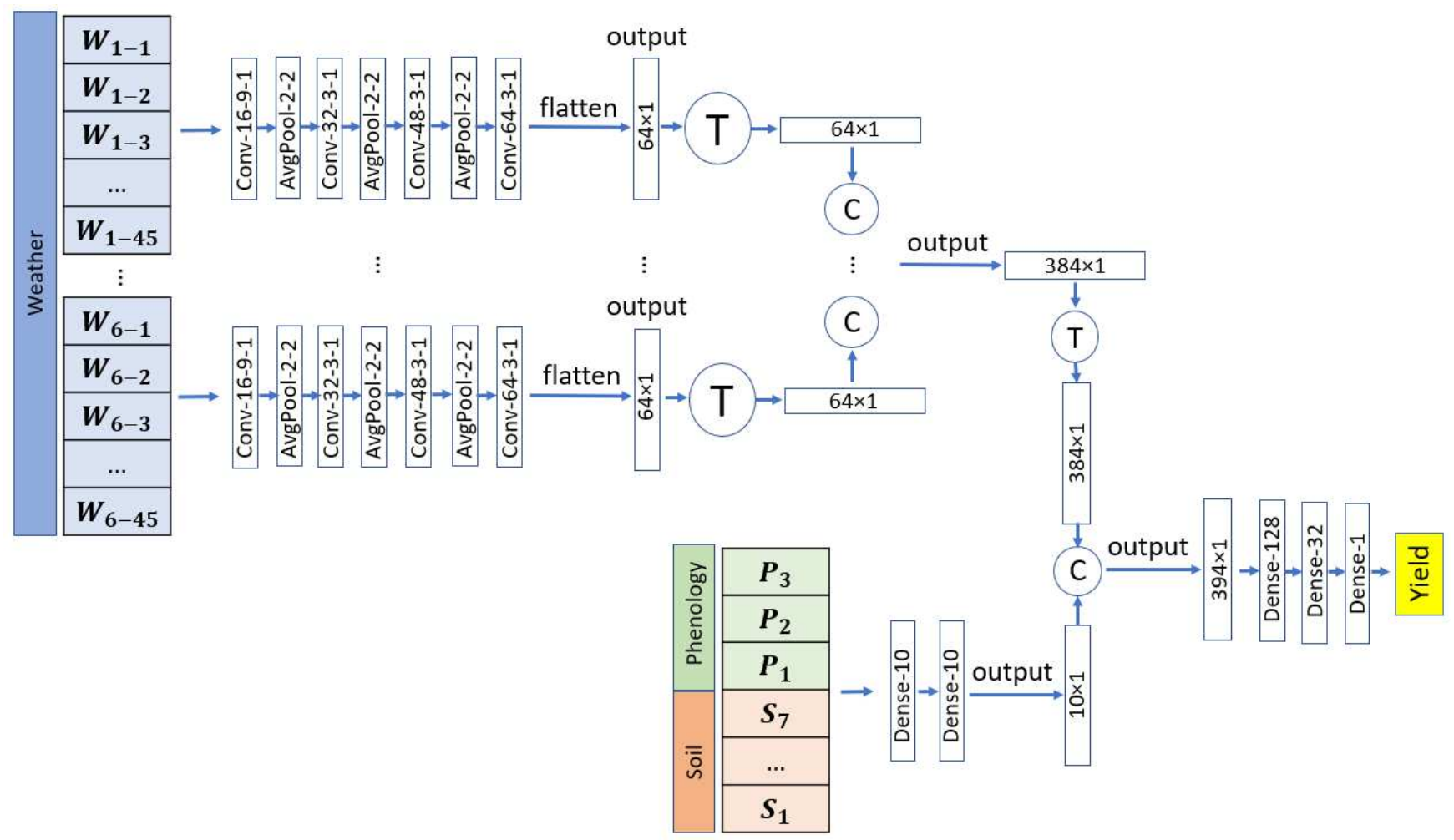

Figure 3. Network architecture of the proposed CNN. The parameters of the convolutional layers are shown as "(convolution type - number of filters - kernel size - stride size)". Conv, AvgPool, Dense, stands for standard convolutional, average pooling, and fully connected layers respectively. The padding type is "valid" for all layers. (C) shows matrix concatenations and (T) refers to matrix transpose. ReLU activation function is used in all layers except the last layers.

\section{Random forests (RF)}

RF includes multiple individual trees and uses Breiman's "bagging” idea to ensemble many decision trees into a single but strong model ${ }^{25}$. It uses the self-help method (i.e., the bootstrap resampling technology) to generate new training sample sets from the original training samples of $\mathrm{N}$ by repeatedly selecting random $\mathrm{k}$ sets of samples. During the overall selecting process, some samples may be collected more than once. The training sample is used to generate $\mathrm{k}$ buffeting the decision or regression trees (CART) for the development of random forests, and then classify the test sample by majority vote decision or use the average as return values. Given the fact that randomness can effectively reduce model variance, random forests, in general, can achieve good generalization ability and low variance resistance without additional pruning.

\section{k-Nearest Neighbor ( $k N N)$}

The k-nearest neighbor $(\mathrm{kNN})$ method is one of the most widely used methods in data mining algorithms in the research community. The method is a long-established non-parametric classifier, introduced by ${ }^{26}$. This method is easy to implement, and it is also simple, efficient, intuitive, and competitive. Therefore, it can be used for both classification and regression problems. At the stage of classification for a given test sample, the kNN algorithm directly searches all training examples by computing the distances between the testing sample and all training data to determine the nearest neighbors and produce the classification 
output. The Euclidean distance function (Equation 1), one of the most widely used distance metric functions in $\mathrm{kNN}^{27}$, was used in this study. The Euclidean distance equation can be represented as Equation 1 where $\mathrm{n}$ is the total number of data points.

$$
d(p, q)=\sqrt{\sum_{i=1}^{n}\left(q_{i}-p_{i}\right)^{2}}
$$

In this formula, $d$ is the Euclidean distance, $p$ and $q$ are the data points consisting of $\mathrm{N}$ dimensions and $i$ is an index number. In addition to the advantages described above, the kNN method has some disadvantages. The kNN method can take a long time to execute when the training dataset is large. The reason is that each query sample is calculated singularly within each training dataset. Moreover, the kNN algorithm is highly sensitive to redundant and irrelevant features and therefore the quality of the features selection must be done carefully. Otherwise, incorrect classification occurs ${ }^{28}$.

\section{LASSO and Ridge Regression}

Different variations of linear regression models (Polynomial, Quantile, Elastic Net, Poison, Negative Binomial, Ordinal, Principal Components, Lasso, Ridge, etc) have successfully been used for creating robust prediction models with comparable performance to non-linear models ${ }^{29}$. Lasso and Ridge Regression are two distinct methods of reducing the variance of linear regression models by shrinking their coefficients. The least absolute shrinkage and selection operator (LASSO) is a regularization method that can exclude some variables by setting their coefficient to zero ${ }^{30}$ and in this manner, Lasso seems like a feature selection algorithm. L1 regularization is added to the linear regression model in LASSO which can shrink coefficients towards zero. On the other hand, Ridge regularization (L2 regularization), although reduces coefficients toward zero, never sets any of them as zero ${ }^{31}$.

\section{Regression Tree (RT)}

A tree is created by splitting the root node of the tree into various partitions that constitute the successor nodes. The process is repeated recursively on each partition known as binary recursive partitioning ${ }^{32}$. Initially, all data points are partitioned into the same group. The algorithm begins partitioning the data into binary partitions/branches. Among all the possible binary splits in each of the tree levels, the algorithm selects the split that minimizes the sum of the squared differences from the mean in the two separate partitions. This splitting algorithm is implemented on every new branch. The process continues until each node becomes a terminal node. A terminal node is a node that has a user-specified minimum node size or if the sum of squared difference from the mean in that node is zero (whichever is reached earlier in the tree).

\section{Support Vector Regression (SVR)}

SVR uses the support vector machine (a classification algorithm) to predict a continuous variable. SVR tries to fit the best line within a threshold of values (epsilon-insensitive tube) ${ }^{33}$. This tube contains the margin of error the model can have. The distance between the data points and the tube is measured and labeled as the support vectors. The objective function of the optimization problem is (Equation 2)

$$
\operatorname{Min} \frac{1}{2}\|w\|^{2}+C \sum_{i=1}^{n}\left|\varepsilon_{i}\right|
$$

Where $w$ stands for the distance between the support vectors, $C$ is a trade-off parameter defined by the user and $\varepsilon_{i}$ is the margin of error.

\section{XGBoost}

It is an implementation of gradient boosted decision trees designed for speed and performance. XGBoost stands for extreme gradient boosting (improved version of gradient boosting machine) and its inception dates back to a research project by Tianqi Chen in $2016^{34}$. XGBoost is an ensemble of decision trees; it is a sequential build-off from various decision trees where each tree works to enhance the performance of the prior tree. In XGboost, the training of each tree is parallelized and this significantly boosts the training speed. XGBoost has been widely used for solving machine learning problems in crop yield.

\section{Deep neural networks (DNN)}

Deep neural networks belong to the class of representation learning methods with multiple levels of abstraction, which can learn the underlying representation of data without the need for handcrafted features ${ }^{35}$. Representation learning is a set of methods that allows a model to be fed with raw data and to automatically discover the relationships between inputs and outputs ${ }^{13}$. The DNN model that has been used in this paper is a feedforward neural network that has multiple hidden layers. Deep neural networks apply a nonlinear function to the output of each hidden layer which makes them highly nonlinear. DNN models 
are trained with gradient-based optimization methods to minimize the desired error function for the task for which they are used. DNN models have recently been used for crop yield prediction which has shown great success by outperforming other traditional machine learning methods ${ }^{13,16,36-38}$

The traditional neural networks with a single hidden layer have also been widely used to estimate FC and PWP ${ }^{39,40}$. This method is a mathematical model developed by the inspiration of the structure of the biological brain ${ }^{41}$. The method is a supervised model that has skills to learn from a training dataset and store the pattern of the data as a weighted connection of nodes. After the learning process, when the new data is applied to the ANN model, it recognizes the pattern from the data and classifies it. In this way, the method gives results quickly and accurately ${ }^{42}$.

\section{Model interpretability}

Recently, there has been a huge inclination toward applying interpretation tools on tree-based ensemble models (e.g. Random Forest and Gradient Boosting Trees) for developing yield prediction models ${ }^{12,15,43}$. In general, tree-based ensemble models lead to more accurate results compared to other baseline machine learning models, such as linear regression. However, the black-box nature of the ensemble models does not provide the reason (features) that are important in determining the crop yield values and whether these features are reducing or increasing the yield values. It is vital to maintain a trade-off between model accuracy and interpretability. Therefore, in this study, SHAP, and Shapley values are applied to provide an interpretable measure for the XGboost model. In this way, the model achieves both an optimal accuracy and decent interpretability ${ }^{44}$. Shapley values were created based on the game theory ${ }^{45}$. Shapley values show the contribution of each feature in the model's outcome. In computing the Shapley values, the mean marginal effect of feature values on the prediction results is analyzed by evaluating feature permutations for all the features to calculate their associate Shapley values. More important features are assigned with greater Shapley values due to their higher contribution to the model results. In the literature, there are several methods used for estimating Shapley values, one of which is called SHAP (Shapley Additive exPlanations) which was introduced by Lundberg and Lee as the most efficient and theoretically optimal way of calculating Shapley values in 2016. This paper uses a slight variation of SHAP called TreeSHAP ${ }^{46,47}$, as a robust and efficient technique for calculating Shapley values in tree-based models such as gradient boosted trees.

\section{Input combinations and parameter calibration}

A combination of weekly meteorological variables, crop phenological variables (sowing, flowering, and harvest dates), and soil variables for 271 counties from 1999 to 2019 was used in the study. The meteorological variables included the maximum and minimum temperature, precipitation, solar radiation, humidity level, and wind speed. The soil variables included the volumetric (\%) crop available water at permanent wilting point (LL), field capacity (DUL), and at the saturation point (SAT), bulk density (BD), percentage of sand, silt, and clay. This results in a total of 5692 instances and 280 column features. To reach the most optimized parameter setting for each model, a combination of numerous parameter domain values was tested on the validation set to reach the best parameter that results in the lowest test error. The validation dataset is limited to the data from 1999 to 2016. The best parameters setting is chosen by either using a grid search cross-validation or randomized search cross-validation with 1000 iterations on a 3-fold cross-validation setting (3000 trials for each model). The range of tested values for parameters is chosen based on the domain knowledge. The tested parameters and the resulted best parameter estimation are shown in Table 2 .

We plotted the ground truth yield and the predicted yield by the XGboost model in Figure 4 to see how close the prediction results are to the ground truth values. As shown in Figure 4, the predicted results of the XGboost and DNN models and the ground truth values have a significantly stronger association compared to the other models. The correlation coefficient of their relationships is 0.71 and 0.70 respectively. In other words, about 50 percent of the variability in the ground truth values can be explained by the predicted results of the XGboost model $\left(R^{2}=0.49\right)$. As it is observed in the graph, the range of predicted yield for the SVR model is very narrow which means that SVR model predicts most of the instances to be between 6 and 7 and cannot be a reliable predictor for yield values.

\section{Model evaluation}

The performance of models was evaluated using mean absolute error (MAE) (Equation 3), root mean square error (RMSE) (Equation 4), and correlation coefficient $r$ (Equation 5):

$$
\begin{aligned}
& M A E=\frac{\sum_{i=1}^{n}\left|y_{i}-\hat{y}_{i}\right|}{n} \\
& R M S E=\sqrt{\frac{\sum_{i=1}^{n}\left(y_{i}-\hat{y}_{i}\right)^{2}}{n}}
\end{aligned}
$$




\begin{tabular}{lll}
\hline Model & Parameters & Best Parameter \\
\hline SVR & C & 1 \\
& Gamma & 0.01 \\
& Kernel type & Rbf \\
& Epsilon & 0.15 \\
\hline KNN-regression & Leaf size & 45 \\
& Number of neighbors & 3 \\
& P & 1 \\
\hline Lasso regression & alpha & 0.0001 \\
\hline Ridge regression & alpha & 0.01 \\
& Solver & Auto \\
\hline Regression tree & Criterion & Mse \\
& Maximum depth & 8 \\
\hline Random Forest & Number of estimators & 644 \\
& Max. feature numbers & Sqrt \\
& Max. depth & 57 \\
& Min. samples split & 5 \\
& Min. samples leaf & 1 \\
& Bootstrap & FALSE \\
\hline XGBoost & Max. depth & 11 \\
& Objective & {$[$ reg:squared error] } \\
& regularization alpha & 0.0001 \\
& Min. child weight & 5 \\
& Gamma & 0.05 \\
& Learning rate & 0.09 \\
& Booster & Gbtree \\
& Subsample & 0.6 \\
& Column sample by tree & 0.9 \\
\hline Number of layers & 5 \\
& Number of neurons & 50 \\
& Optimizer & Adam \\
& Batch size & 8 \\
& Number of epochs & 50 \\
& Learning rate & ReLu \\
& & 0.0003 \\
\hline & & \\
& & \\
& &
\end{tabular}

Table 2. Parameter settings for the machine learning models used for predicting the winter wheat yield. 

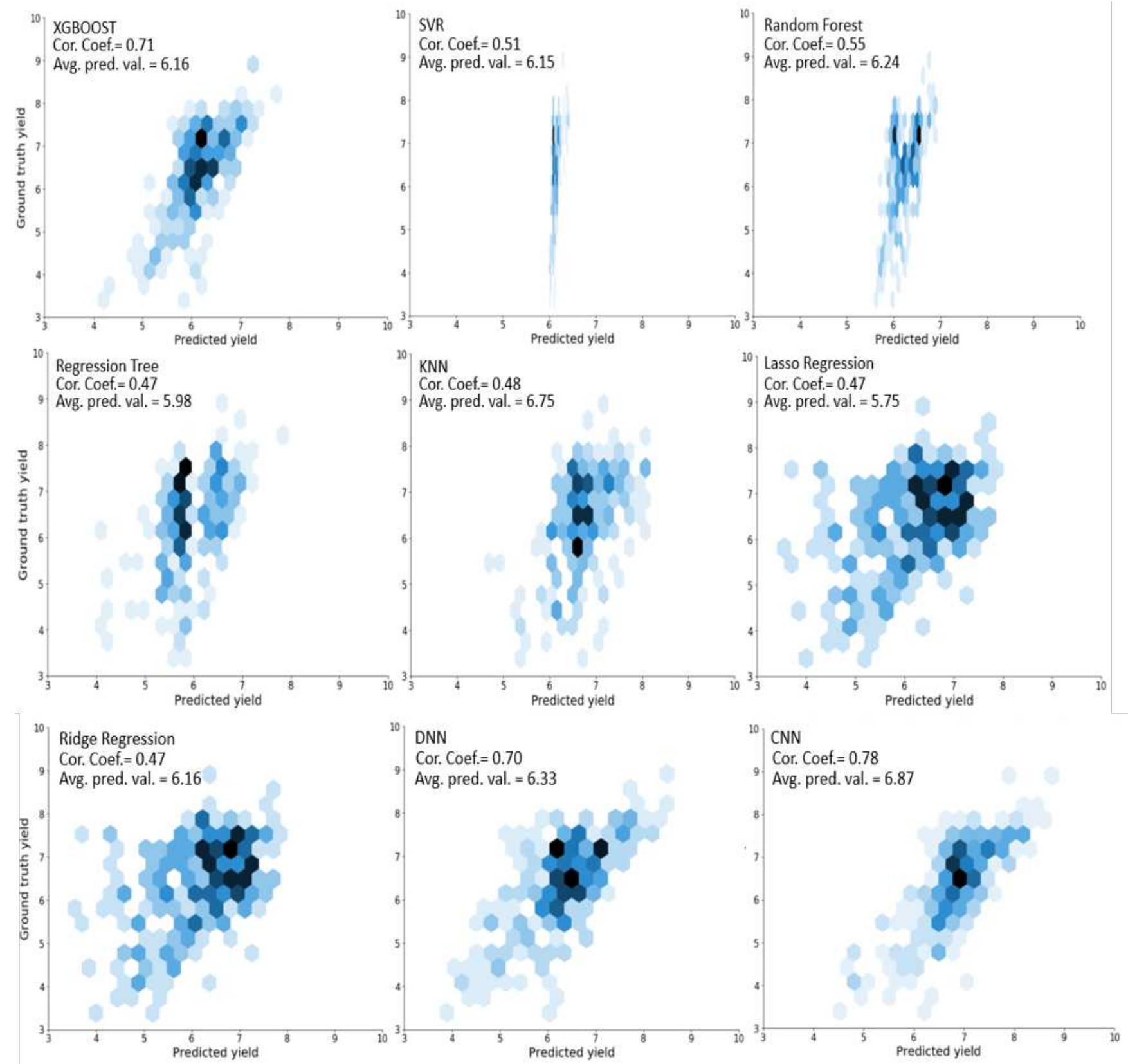

Figure 4. Scatter plot of the predicted winter wheat yield vs. ground truth yield values for the eight models. 


$$
r=\sqrt{1-\frac{\sum_{i=1}^{n}\left(y_{i}-\hat{y}\right)^{2}}{\sum_{i=1}^{n}\left(y_{i}-\bar{y}\right)^{2}}}
$$

$\hat{y}_{i}, \bar{y}_{i}$ and $n$ stand for the ground truth and predicted values and the total number of data points respectively.

\section{Results}

\section{Prediction results}

Using the best parameter settings achieved in "Model interpretability" section, three models are trained for each of the machine learning methods in the study. One model is trained on the data between 1999 to 2016 and tested on the data of 2017, the other model is trained using the data between 1999 to 2017 and tested in 2018, and the last model is trained on the data between 1999 to 2018 and tested on the data of 2019. The train and test results based on the model evaluation metrics described in "Input combinations and parameter calibration" section are shown in Table 3.

\begin{tabular}{llllllllll}
\hline & KNN & Rand. For. & XGB & Lasso & Ridge & Reg. Tree & SVR & DNN & Proposed \\
\hline Training 1999 to 2016 (RMSE) & 0.33 & 0.04 & 0.18 & 0.57 & 0.56 & 0.46 & 0.33 & 0.41 & 0.48 \\
Test on 2017 (RMSE) & 0.88 & 0.8 & 0.85 & 1.19 & 1.05 & 0.85 & 0.86 & 0.8 & 0.66 \\
Training 1999 to 2017 (RMSE) & 0.38 & 0.27 & 0.31 & 0.61 & 0.62 & 0.54 & 0.38 & 0.44 & 0.47 \\
Test on 2018 (RMSE) & 1.06 & 1.13 & 0.94 & 1.23 & 1.39 & 0.95 & 1.04 & 1.00 & 0.84 \\
Training 1999 to 2018 (RMSE) & 0.44 & 0.39 & 0.39 & 0.67 & 0.71 & 0.59 & 0.44 & 0.43 & 0.5 \\
Test on 2019 (RMSE) & 1.07 & 0.92 & 0.87 & 1.27 & 1.69 & 0.98 & 1.02 & 0.74 & 0.64 \\
Training 1999 to 2016 (MAE) & 0.25 & 0.03 & 0.14 & 0.44 & 0.44 & 0.35 & 0.24 & 0.32 & 0.38 \\
Test on 2017 (MAE) & 0.67 & 0.66 & 0.68 & 1.03 & 0.88 & 0.69 & 0.71 & 0.66 & 0.52 \\
Training 1999 to 2017 (MAE) & 0.27 & 0.18 & 0.22 & 0.47 & 0.48 & 0.41 & 0.27 & 0.35 & 0.37 \\
Test on 2018 (MAE) & 0.88 & 0.9 & 0.77 & 1.03 & 1.16 & 0.78 & 0.82 & 0.81 & 0.71 \\
Training 1999 to 2018 (MAE) & 0.3 & 0.25 & 0.27 & 0.51 & 0.55 & 0.44 & 0.29 & 0.34 & 0.39 \\
Test on 2019 (MAE) & 0.84 & 0.75 & 0.69 & 1.00 & 1.38 & 0.81 & 0.85 & 0.59 & 0.5 \\
Training 1999 to 2016 (r) & 0.94 & 1 & 0.98 & 0.81 & 0.82 & 0.89 & 0.94 & 0.91 & 0.9 \\
Test on 2017 (r) & 0.3 & 0.47 & 0.54 & 0.41 & 0.43 & 0.57 & 0.35 & 0.6 & 0.65 \\
Training 1999 to 2017 (r) & 0.92 & 0.97 & 0.95 & 0.78 & 0.77 & 0.83 & 0.92 & 0.9 & 0.88 \\
Test on 2018 (r) & 0.3 & 0.15 & 0.47 & 0.15 & 0.03 & 0.48 & 0.28 & 0.39 & 0.78 \\
Training 1999 to 2018 (r) & 0.89 & 0.93 & 0.92 & 0.73 & 0.7 & 0.8 & 0.9 & 0.9 & 0.87 \\
Test on 2019 (r) & 0.49 & 0.5 & 0.64 & 0.44 & 0.46 & 0.57 & 0.45 & 0.73 & 0.81 \\
\hline
\end{tabular}

Table 3. Training and validation results (RMSE and $r$ values) for the machine learning models used for predicting winter wheat yield. Rand. For. stands for Random forest and Reg. Tree stands for Regression Tree.

Based on the results shown in this Table 3, our proposed model outperformed other methods to a varying extent due to its highly nonlinear modeling structure as well as capturing the temporal dependencies of weather data. XGboost and DNN had a comparable performance while DNN was slightly better than XGboost. DNN outperformed all other models concerning all performance measures except the XGboost model as the DNN model is highly nonlinear and automatically discovers the relationship between the input data and the yield by extracting relevant features from the input data. The linear models such as Lasso and Ridge had a weak performance compared to other models due to their linear modeling structure which fails to capture the nonlinear effects of weather and soil conditions. XGboost performance is better than random forest and regression tree models due to the use of gradient boosted trees which significantly improves its performance. The prediction error for winter wheat yield compared to the ground truth yield data (i.e., the observed yield) across the 271 counties varies from $0.1 \%$ to 67.9\% in the year 2019 (Figure 5). KNN and SVR models also had a comparable performance and they had a higher prediction accuracy compared to linear models such as Lasso and Ridge due to capturing nonlinear effects of weather and soil data.

\section{ANALYSIS}

\section{Feature selection}

We have predicted the yield based on weather components and soil conditions. Feature selection is performed on the trained XGboost model to find the relative importance of each feature. Weather and soil data are generally represented by numerous variables, which do not have equal effect or importance in yield prediction. Thus, it is of paramount importance to find important 


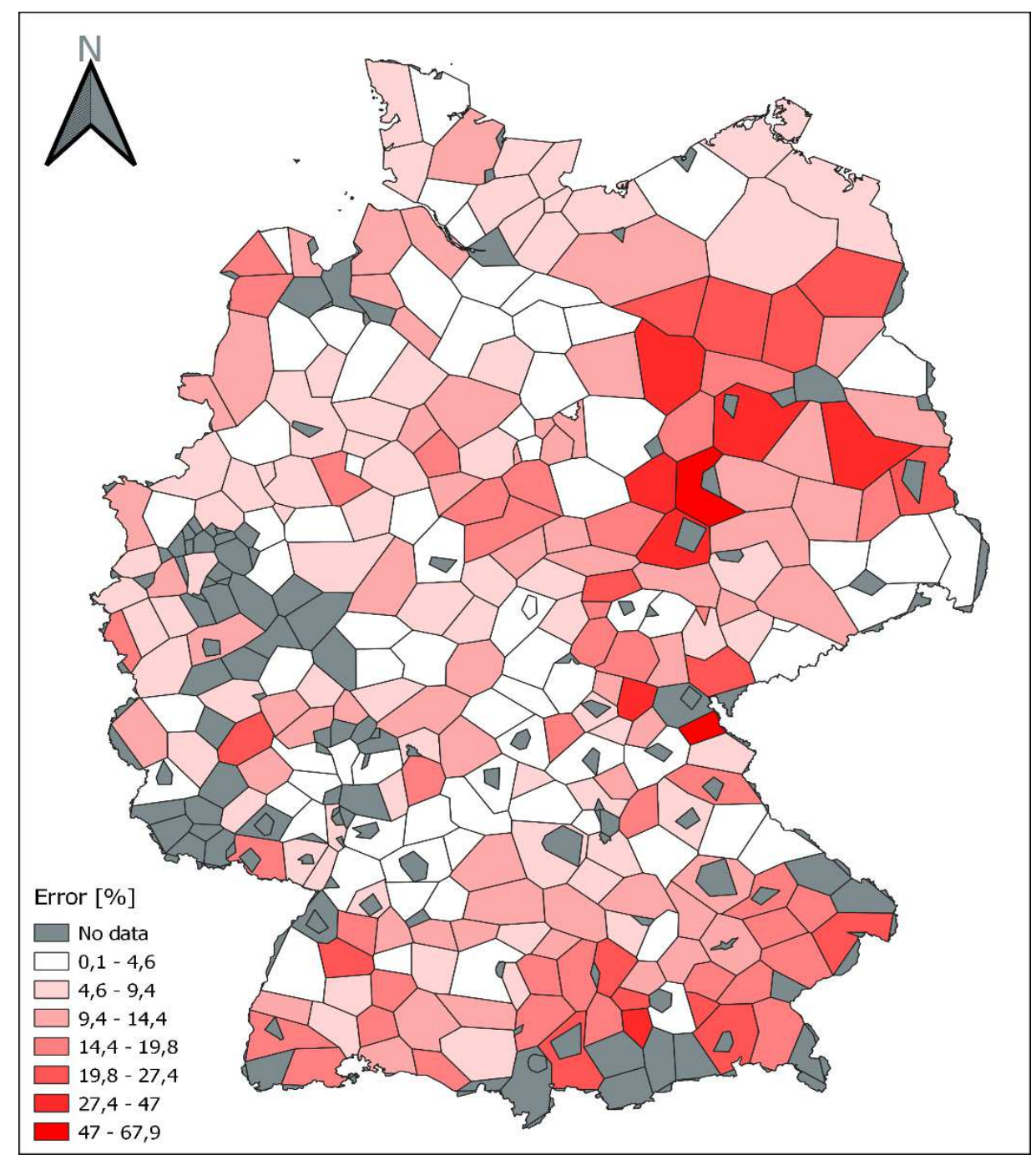

Figure 5. Percentage (\%) error in winter wheat yield predictions using XGBoost model in 271 counties. The counties in dark grey color indicate that ground truth yields were not available for the year 2019. 
variables and remove the redundant variables that might decrease the prediction accuracy. In this paper, we used Shapley values to find input variables that have a higher contribution to the model results. Features with large Shapley values are important. The average absolute Shapley value per feature across the data is calculated to find the global importance. The results are shown in Figure 6 Red bars show that features that have a positive correlation with yield impact (thus positively impact the yield value) and blue bars show the features which have a negative correlation with yield impact (thus negatively impact the yield value).

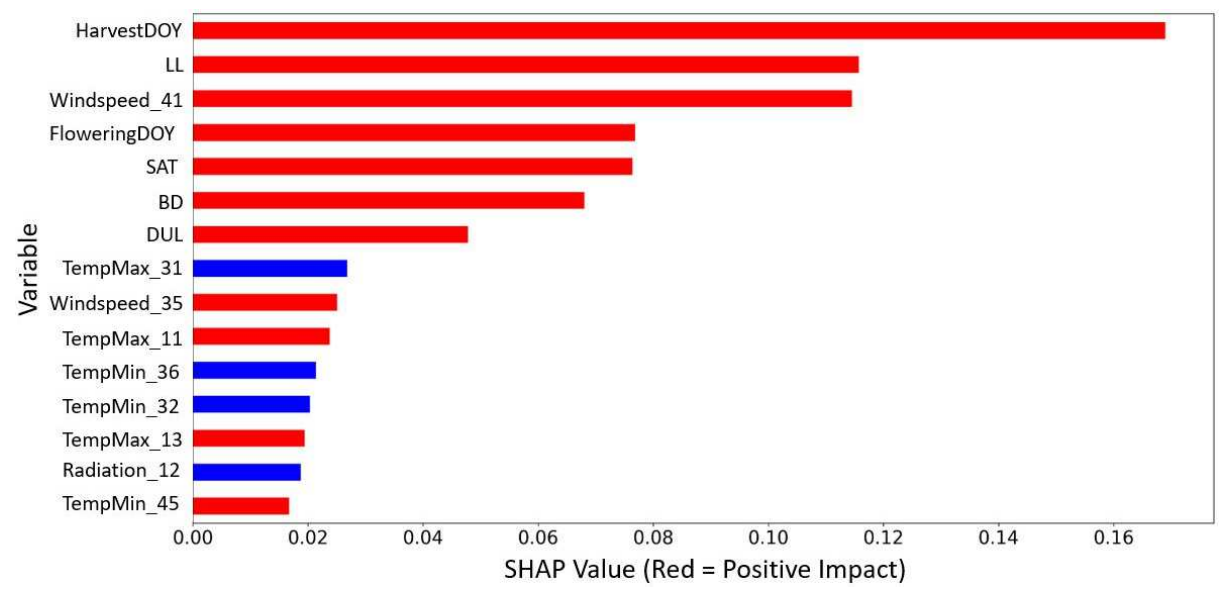

Figure 6. Shapley values for the top 15 features in yield prediction of winter wheat for 2019 (For the weather variables on the vertical axis, the variable name refers to the title of the weather variable plus the week of the year, separated by an underscore sign. (HarvestDOY=Harvest day of the year; $\mathrm{LL}=$ crop available water at permanent wilting point; $\mathrm{SAT}=\mathrm{crop}$ available water at the saturation point; $\mathrm{DUL}=$ crop available water at the field capacity; BD=soil bulk density).

In addition, to further dive into understanding the feature effects and their importance, we have created a series of force plots; the plots for the top three important features (refer to Figure 6) in yield prediction are shown in Figure 7, Figure 8, and Figure 9.

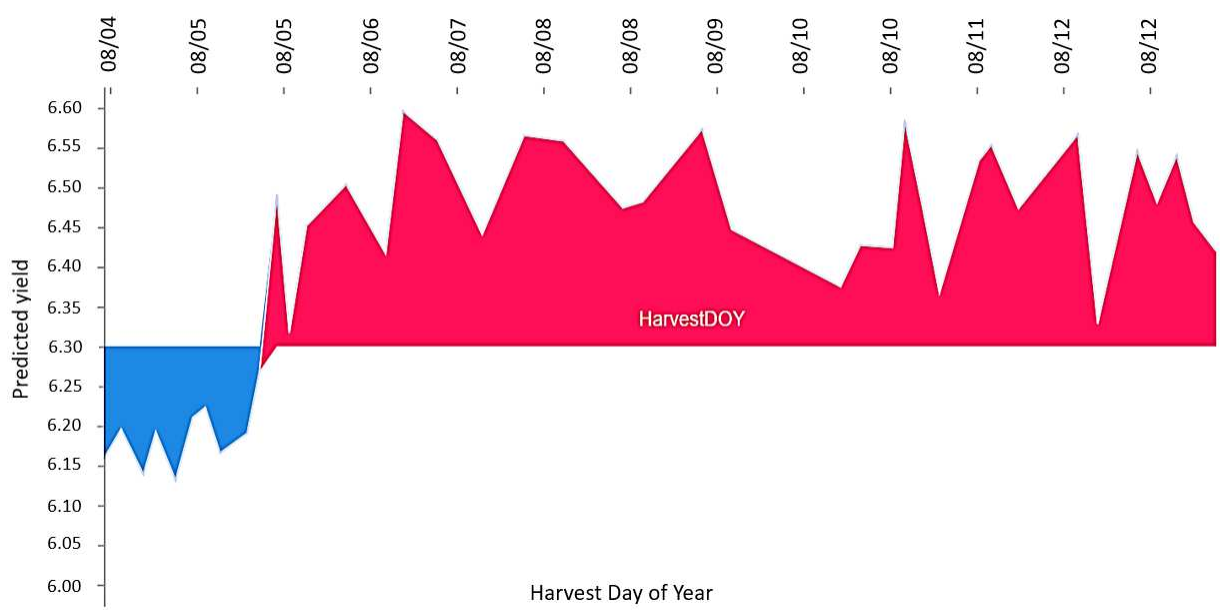

Figure 7. Force plot for Harvest day of the year (HarvestDOY, refer to Figure 6) and predicted yield (in tons ha- 1 ) of the year 2019.

Figure 7, 8, and 9 interpret the contribution of the top three important features in modeling the yield prediction. The vertical axes in these plots show the predicted yield value (in tons ha ${ }^{-} 1$ ), and the horizontal axes refer to the selected features' values. The intercept of the vertical and horizontal axis shows the base value of the prediction results. The base value is the average of yield prediction results. Parts of the plots in red show the range of feature values that positively affect the yield value. For instance, Figure 7 shows that harvesting after the 5th day of August positively contributes to the yield values. Also, Figure 8 shows that LL lower than 0.13 negatively affects the yield values, and LL higher than 0.19 has a significant positive steady effect of boosting yield values. Figure 9 is indeed divided into two parts; wind speed of less than 2.35 in week 41 negatively affects the yield values and vice versa. 


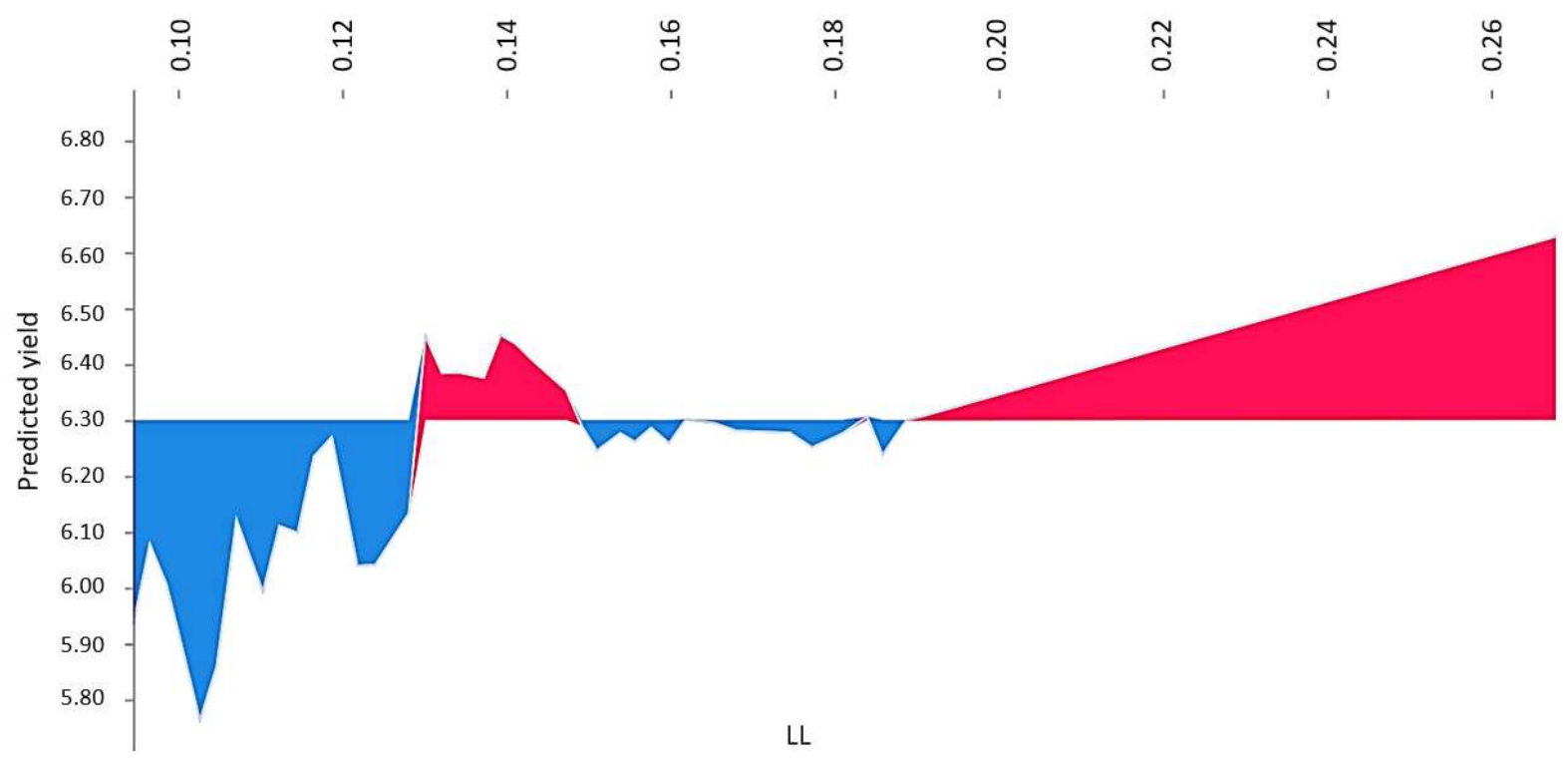

Figure 8. Force plot for volumetric (\%) water at permanent wilting point (LL, refer to Figure 6) and predicted yield (in tons $\mathrm{ha}^{-} 1$ ) in the year 2019.

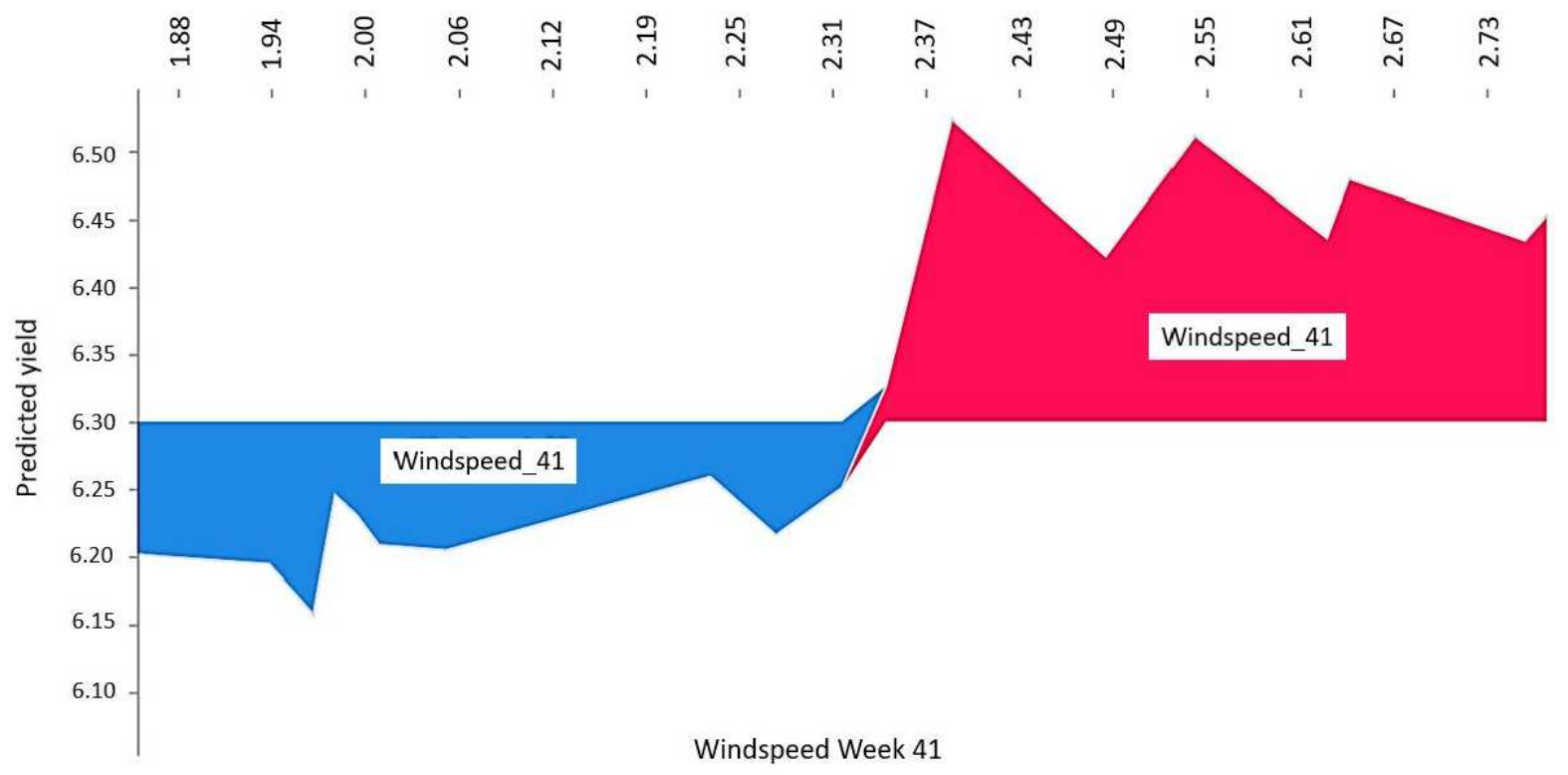

Figure 9. Force plot for wind speed in week 41 (Windspeed 41, refer to Figure 6) and predicted yield (in tons ha ${ }^{-}$) in the year 2019. 
Additionally, three instances with the highest, average, and lowest yield values were selected to interpret the effects of their features on the predicted yield values using Shapley values. Force plots are effective in interpreting the prediction value of the XGboost model in critical instances ${ }^{44}$. The interpretation is conducted using SHAP explanation force plots in Figure 10 part a, $\mathrm{b}$, and $\mathrm{c}$ show the force plots associated with the instances with the lowest, average, and highest yield values respectively. The contribution of each feature to the output value is shown with arrows with their force associated with the Shapley values. Red arrows show features increasing the prediction results (i.e., yield values) to reach the ground-truth value (output value), and blue arrows show the ones decreasing the prediction to reach the same output value. The arrows with positive and negative effects on yield values compensate on a point which is the prediction (output) value. The base value (like Figure 10) is the mean of the ground truth yield values over the entire dataset.

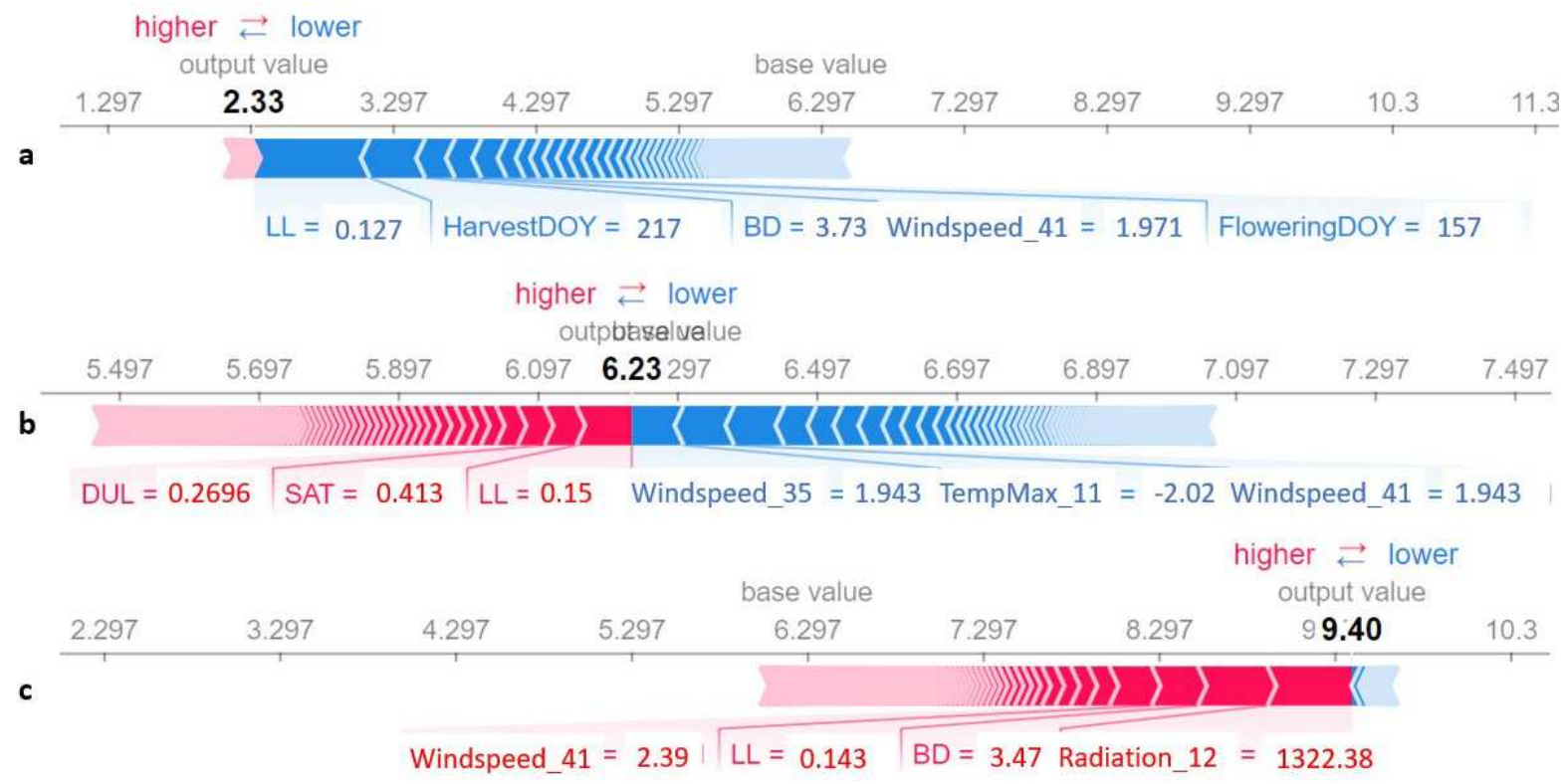

Figure 10. SHAP values to explain the predicted yield values for instances with the minimum (a), average (b), and maximum (c) yield values in 2019.

Figure 12 and 13 provide temporal resolutions that allow the determination of critical periods for a deeper understanding of how the intricate agronomic system functions. Out of the six weather variables examined in Figure 11, the temperature (minimum and maximum) and the precipitation have the highest predictive capacity for the yield prediction shown by the sum of the values of the effects 3.71,3.28, and 3.31 respectively which goes with the findings of earlier studies suggesting that temperature-related indicators have the highest predictive power for crop yields ${ }^{48}$. This is reasonable from an agronomic perspective as it is evident from Figure 11, the higher SHAP values for minimum temperature compared to the other weather components around week 36-37 which coincide with the reproductive phase, the most sensitive growth stage to the low temperatures. Additionally, higher SHAP values of minimum temperature at the start of the crop growth period (1-2 weeks) can also affect the crop yield prediction. Several studies have reported that increased minimum temperature reduced the cold injury and frost damage to seedlings ${ }^{34,49}$ leading to increased crop yield and warming-induced enhancement of grain weight ${ }^{50}$. The maximum temperature was found most sensitive in yield prediction around week 31 (coinciding with the flowering phase), and around week 36-37 coinciding with the reproductive phase. From an agronomic perspective, the higher maximum temperature can negatively affect winter wheat yield by reducing grain number and grain size ${ }^{51,52}$, and shortening the duration of wheat grain filling ${ }^{53}$. Furthermore, the negative yield effects of high temperatures are also associated with the water and heat stress conditions during the sensitive crop growth stages mainly leading to a reduction in photosynthesis rate, increased respiration, accelerated leaf senescence, and enhanced evapotranspiration finally hampering the grain numbers as well ${ }^{54}$. Precipitation holds an equally important predictive skill for the crop yield as well in the current study and could be attributed to the fact that under less precipitation, crops exposed to drought stress especially during reproduction, grain yields can be negatively affected due to hampered nutrients uptake, and in combination with a diminished surface cooling, crop canopy temperatures shoot up and lead to further decrease in photosynthetic rates ${ }^{55}$. Higher SHAP values of the Precipitation around weeks 21-23, a period that could be linked to the stem elongation, compared to all the other weather variables indicated higher predictive skills of winter wheat crop yield as stem elongation is a critical phase for yield formation and water is needed for expansive growth processes of the crop that bring up the spike to the top of the canopy through the unfolding leaf, as well as for spike growth and 
the cell expansion, pollen ripening or grain growth and filling ${ }^{56}$. In terms of soil variables (refer to Figure 10), our analysis showed LL, SAT, BD, and DUL to be sensitive to yield prediction. This goes in line with the findings of Peichl et al. $(2021)^{57}$ indicating soil moisture to be the most important variable for winter wheat yield prediction. All of these factors are known to affect crop available water which has direct consequences on the growth and development. For instance, sandy soils which are having a lower water holding capacity may result in a water shortage for the winter wheat growth ${ }^{58}$. On the other hand, excessive water in the soil (waterlogged conditions) could lead to aeration stress and thereby damaging the plants resulting in yield losses ${ }^{59}$. It is also being reported that excessive soil water encourages the development of pathogens and hurdles the necessary management operations ${ }^{60}$.
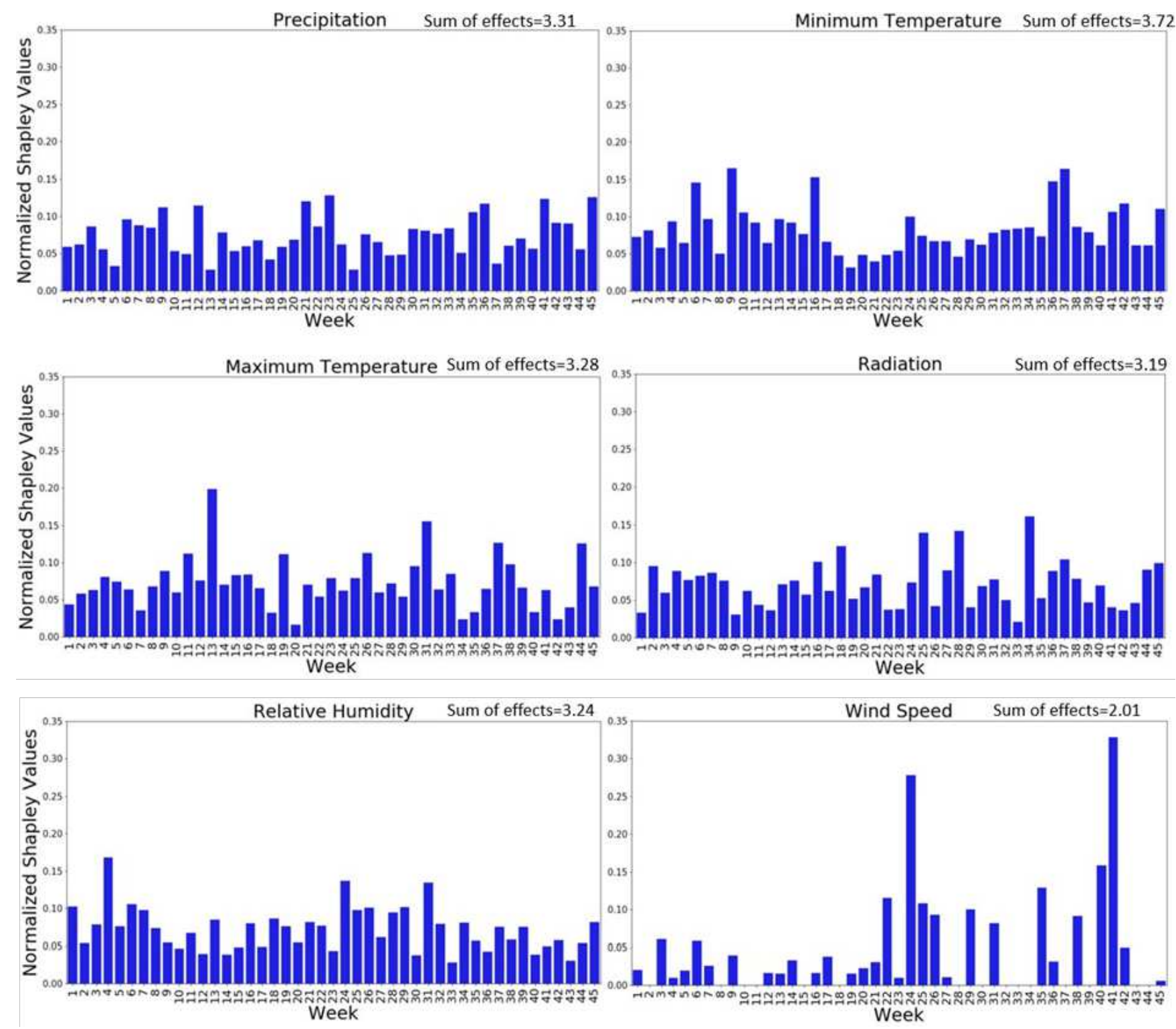

Figure 11. Estimated effects of six weather components on winter wheat measured for 45 weeks of 2019, starting from late Sept (week 1) and ending in mid-August (week 45). The vertical axes were normalized across all-weather components to make the effects comparable.

\section{Generalization Power of the XGboost Model}

To evaluate the performance of the feature selection method, we obtained prediction results based on a subset of features. As such, we sorted the all features based on their estimated effects and selected 75 percent and 50 percent of the most important components in the data. Table 4 shows the yield prediction performance of the XGboost model using these selected features. 


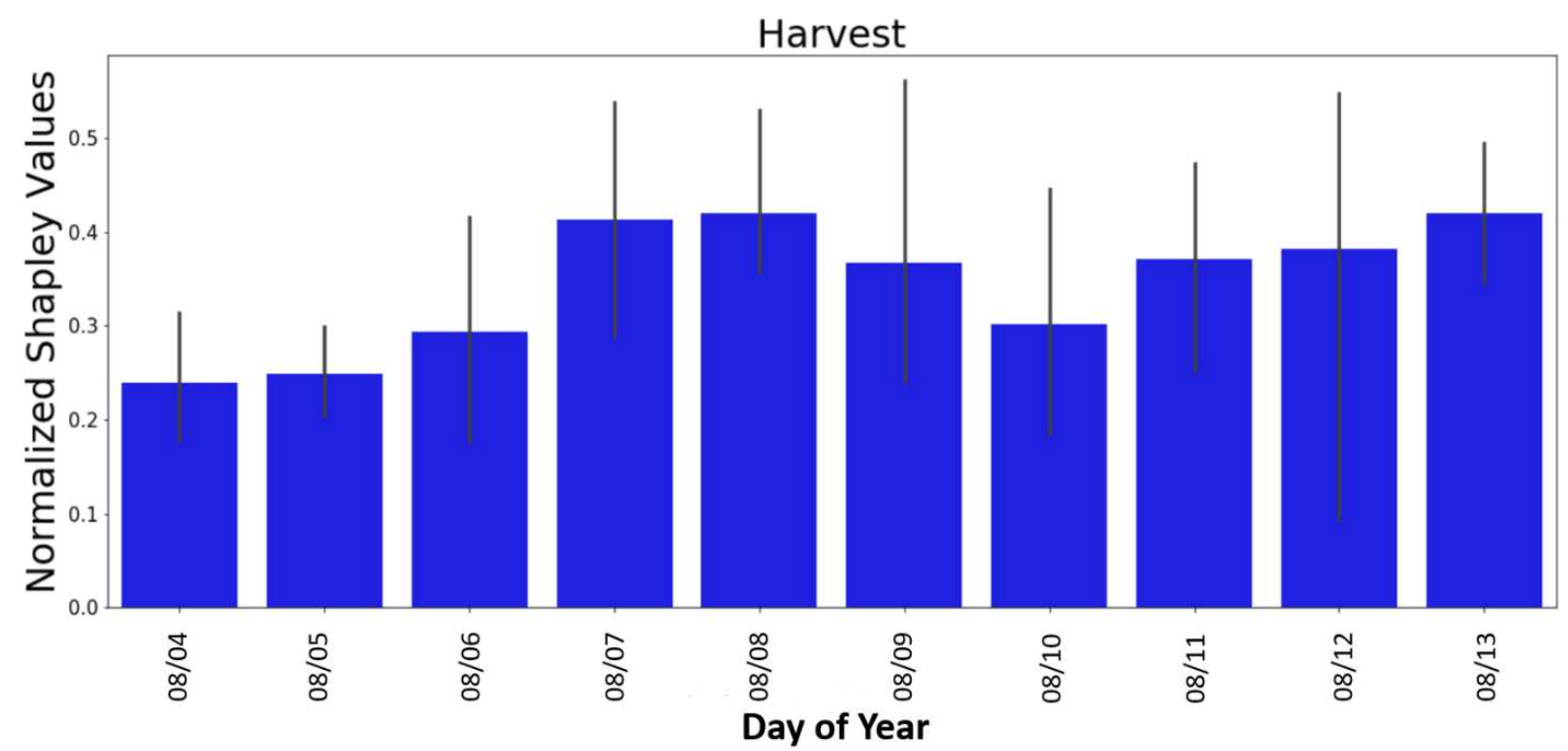

Figure 12. Estimated effects of harvesting date on the yield in 2019. The vertical axes are normalized across harvesting dates to make the effects comparable.

Flowering

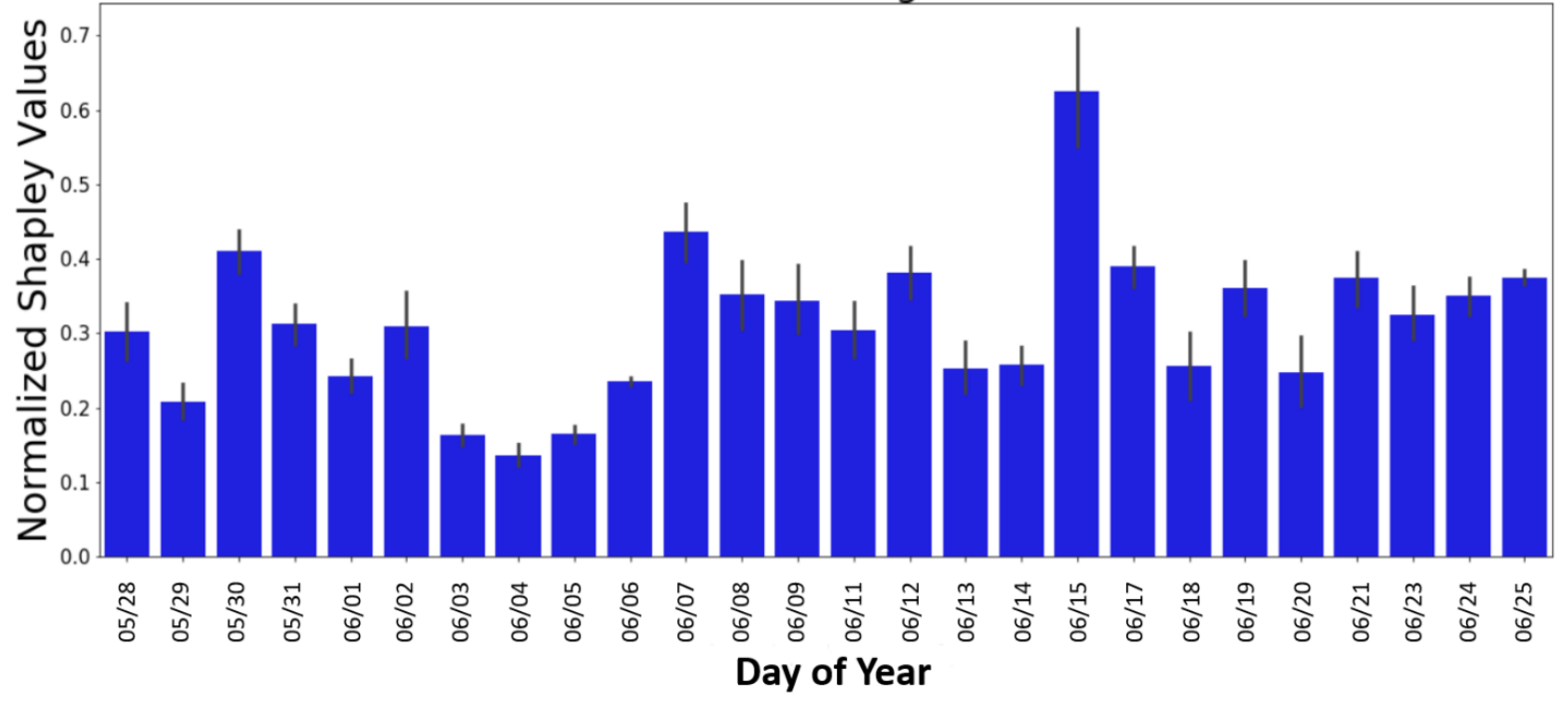

Figure 13. Estimated effects of the flowering date on the yield in 2019. The vertical axes are normalized across flowering dates variable to make the effects comparable. 
The prediction accuracy of XGboost not only did not drop significantly compared to the corresponding results in Table 4 but also got slightly improved which shows that the feature selection method can effectively find the important features. In addition to evaluating the difference between the prediction results of soil and weather variables separately, the XGboost model was trained using only the Weather and Soil variables separately. As expected from Table 4, soil variables are more important features for yield prediction compared to the weather variables.

\begin{tabular}{llllll}
\hline & $\begin{array}{l}\text { XGboost } \\
\text { (Full Model) }\end{array}$ & $\begin{array}{l}\text { XGboost) } \\
\text { (top 75\%) }\end{array}$ & $\begin{array}{l}\text { XGboost } \\
\text { (top 50\%) }\end{array}$ & $\begin{array}{l}\text { XGboost } \\
\text { (Weather) }\end{array}$ & $\begin{array}{l}\text { XGboost) } \\
\text { (Soil) }\end{array}$ \\
\hline Test on 2017 (RMSE) & 0.85 & 0.85 & 0.78 & 1.2 & 0.76 \\
Test on 2018 (RMSE) & 0.94 & 1.03 & 0.97 & 1.11 & 1.03 \\
Test on 2019 (RMSE) & 0.87 & 0.79 & 0.78 & 1.15 & 0.93 \\
Test on 2017 (MAE) & 0.68 & 0.68 & 0.64 & 1.04 & 0.6 \\
Test on 2018 (MAE) & 0.77 & 0.84 & 0.79 & 0.9 & 0.8 \\
Test on 2019 (MAE) & 0.69 & 0.61 & 0.62 & 0.95 & 0.74 \\
Test on 2017 (Corr) & 0.54 & 0.58 & 0.68 & 0.35 & 0.47 \\
Test on 2018 (Corr) & 0.47 & 0.42 & 0.57 & 0.01 & 0.37 \\
Test on 2019 (Corr) & 0.64 & 0.69 & 0.71 & 0.5 & 0.46 \\
\hline
\end{tabular}

Table 4. Test results of using the different combination of features for predicting winter wheat yield with the XGboost model

\section{Conclusions}

In this paper, we compared the performances of eight different machine learning and deep learning methods for winter wheat yield prediction based on weather, soil, and crop phenology data. We also proposed a CNN model for winter wheat forecasting which outperformed other models. The machine learning methods included KNN, random forest, XGboost, regression tree, Lasso, Ridge, SVR, and DNN. Our results indicated that nonlinear models such as DNN and XGboost outperform other methods to a varying extent because the crop yield is a highly complex trait that depends on the many interactive factors such as genotype and environmental conditions. As a result, nonlinear models are more effective in finding the functional relationship between the crop yield and input data. The results also revealed that the deep neural networks often a higher prediction accuracy than XGboost. The linear models such as Lasso and Ridge regression did not perform well due to their linear modeling structure which cannot capture the nonlinear effects of weather and soil variables. One of the main limitations of machine learning models is their black box property. As a result, we moved beyond prediction and performed feature selection, as it provided key results towards explaining yield prediction (variable importance by time period). The feature selection method estimated the individual effect of weather components, soil conditions, and phenology variables as well as the time period that these variables become important. As such, our study shows which variables have the most significant effect on winter wheat yield.

\section{References}

1. Konduri, V. S., Vandal, T. J., Ganguly, S. \& Ganguly, A. R. Data Science for Weather Impacts on Crop Yield. Front. Sustain. Food Syst. 4, 52, DOI: 10.3389/fsufs.2020.00052 (2020). Publisher: Frontiers.

2. Xu, X. et al. Design of an integrated climatic assessment indicator (ICAI) for wheat production: A case study in Jiangsu Province, China. DOI: 10.1016/J.ECOLIND.2019.01.059 (2019).

3. Moeinizade, S., Hu, G., Wang, L. \& Schnable, P. S. Optimizing Selection and Mating in Genomic Selection with a Look-Ahead Approach: An Operations Research Framework. G3: Genes, Genomes, Genet. 9, 2123-2133, DOI: 10.1534/g3.118.200842 (2019). Publisher: G3: Genes, Genomes, Genetics Section: Genomic Prediction.

4. Basso, B. \& Liu, L. Chapter Four - Seasonal crop yield forecast: Methods, applications, and accuracies. In Sparks, D. L. (ed.) Advances in Agronomy, vol. 154, 201-255, DOI: 10.1016/bs.agron.2018.11.002 (Academic Press, 2019).

5. Khoda Bakhshi, A. \& Ahmed, M. M. Real-time crash prediction for a long low-traffic volume corridor using correctedimpurity importance and semi-parametric generalized additive model. J. Transp. Saf. \& Secur. 0, 1-35, DOI: 10.1080/ 19439962.2021.1898069 (2021). Publisher: Taylor \& Francis_eprint: https://doi.org/10.1080/19439962.2021.1898069.

6. Günay, E. E., Okudan Kremer, G. E. \& Zarindast, A. A multi-objective robust possibilistic programming approach to sustainable public transportation network design. Fuzzy Sets Syst. DOI: 10.1016/j.fss.2020.09.007 (2020). 
7. Haghighat, A. K. et al. Applications of Deep Learning in Intelligent Transportation Systems. J. Big Data Anal. Transp. 2, 115-145, DOI: 10.1007/s42421-020-00020-1 (2020).

8. Parsa, A., Movahedi, A., Taghipour, H., Derrible, S. \& Mohammadian, A. Toward safer highways, application of XGBoost and SHAP for real-time accident detection and feature analysis. Accid. analysis prevention DOI: 10.1016/j.aap.2019.105405 (2019).

9. Chlingaryan, A., Sukkarieh, S. \& Whelan, B. Machine learning approaches for crop yield prediction and nitrogen status estimation in precision agriculture: A review. Comput. Electron. Agric. 151, 61-69, DOI: 10.1016/j.compag.2018.05.012 (2018).

10. Bamney, A. et al. Examining Impacts of COVID-19 Related Stay-at-Home Orders through a Two-way Random Effects Model (2021). Number: TRBAM-21-02358.

11. Mupangwa, W., Chipindu, L., Nyagumbo, I., Mkuhlani, S. \& Sisito, G. Evaluating machine learning algorithms for predicting maize yield under conservation agriculture in Eastern and Southern Africa. SN Appl. Sci. 2, 952, DOI: 10.1007/s42452-020-2711-6 (2020).

12. Shahhosseini, M., Martinez-Feria, R. A., Hu, G. \& Archontoulis, S. V. Maize yield and nitrate loss prediction with machine learning algorithms. Environ. Res. Lett. 14, 124026, DOI: 10.1088/1748-9326/ab5268 (2019). Publisher: IOP Publishing.

13. Khaki, S. \& Wang, L. Crop Yield Prediction Using Deep Neural Networks. Front. Plant Sci. 0, DOI: 10.3389/fpls.2019. 00621 (2019). Publisher: Frontiers.

14. Feng, P. et al. Dynamic wheat yield forecasts are improved by a hybrid approach using a biophysical model and machine learning technique. Agric. For. Meteorol. 285-286, 107922, DOI: 10.1016/j.agrformet.2020.107922 (2020).

15. Kang, Y. et al. Comparative assessment of environmental variables and machine learning algorithms for maize yield prediction in the US Midwest. Environ. Res. Lett. 15, 064005, DOI: 10.1088/1748-9326/ab7df9 (2020). Publisher: IOP Publishing.

16. Van Klompenburg, T., Kassahun, A. \& Catal, C. Crop yield prediction using machine learning: A systematic literature review. Comput. Electron. Agric. 177, 105709, DOI: 10.1016/j.compag.2020.105709 (2020).

17. Khaki, S., Safaei, N., Pham, H. \& Wang, L. WheatNet: A Lightweight Convolutional Neural Network for High-throughput Image-based Wheat Head Detection and Counting. arXiv:2103.09408 [cs] (2021). ArXiv: 2103.09408.

18. Hassan, M. A., Khalil, A., Kaseb, S. \& Kassem, M. A. Exploring the potential of tree-based ensemble methods in solar radiation modeling. Appl. Energy 203, 897-916, DOI: 10.1016/j.apenergy.2017.06.104 (2017).

19. Zhao, G. et al. The implication of irrigation in climate change impact assessment: a European-wide study. Glob. Chang. Biol. 21, 4031-4048, DOI: 10.1111/gcb.13008 (2015)._eprint: https://onlinelibrary.wiley.com/doi/pdf/10.1111/gcb.13008.

20. EUROSTAT. Glossary: Nomenclature of territorial units for statistics (NUTS) (2019).

21. COPERNICUS. CORINE Land Cover (2006).

22. EUROSTAT. NUTS - Nomenclature of territorial units for statistics - Eurostat (2019).

23. Ämter, S. Regionaldatenbank Deutschland (2020).

24. DWD. Wetter und Klima - Deutscher Wetterdienst (2020).

25. Breiman, L. Random Forests. Mach. Learn. 45, 5-32, DOI: 10.1023/A:1010933404324 (2001).

26. Cover, T. \& Hart, P. Nearest neighbor pattern classification. IEEE Transactions on Inf. Theory 13, 21-27, DOI: 10.1109/TIT.1967.1053964 (1967). Conference Name: IEEE Transactions on Information Theory.

27. Hu, L.-Y., Huang, M.-W., Ke, S.-W. \& Tsai, C.-F. The distance function effect on k-nearest neighbor classification for medical datasets. SpringerPlus 5, 1304, DOI: 10.1186/s40064-016-2941-7 (2016).

28. Imandoust, S. B. \& Bolandraftar, M. Application of K-Nearest Neighbor (KNN) Approach for Predicting Economic Events: Theoretical Background. 3, 6 (2013).

29. Safaei, N., Zhou, C., Safaei, B. \& Masoud, A. Gasoline prices and their relationship to the number of fatal crashes on U.S. roads. Transp. Eng. 4, 100053, DOI: 10.1016/j.treng.2021.100053 (2021).

30. James, G., Witten, D., Hastie, T. \& Tibshirani, R. An Introduction to Statistical Learning: with Applications in R. Springer Texts in Statistics (Springer-Verlag, New York, 2013).

31. Hoerl, A. E. \& Kennard, R. W. Ridge Regression: Biased Estimation for Nonorthogonal Problems. Technometrics 12, 55-67, DOI: 10.1080/00401706.1970.10488634 (1970). 
32. Breiman, L., Friedman, J., Charles J. Stone \& Olshen, R. Classification and Regression Trees (Chapman and Hall/CRC, 1984), 1 edn.

33. Cortes, C. \& Vapnik, V. Support-vector networks. Mach. Learn. 20, 273-297, DOI: 10.1007/BF00994018 (1995).

34. Chen, X. \& Chen, S. China feels the heat: negative impacts of high temperatures on China's rice sector. Aust. J. Agric. Resour. Econ. 62, 576-588, DOI: $10.1111 / 1467-8489.12267$ (2018). _eprint: https://onlinelibrary.wiley.com/doi/pdf/10.1111/1467-8489.12267.

35. LeCun, Y., Bengio, Y. \& Hinton, G. Deep learning. Nature 521, 436-444, DOI: 10.1038/nature14539 (2015). Bandiera_abtest: a Cg_type: Nature Research Journals Number: 7553 Primary_atype: Reviews Publisher: Nature Publishing Group Subject_term: Computer science;Mathematics and computing Subject_term_id: computer-science;mathematicsand-computing.

36. Khaki, S., Wang, L. \& Archontoulis, S. V. A CNN-RNN Framework for Crop Yield Prediction. Front. Plant Sci. 0, DOI: 10.3389/fpls.2019.01750 (2020). Publisher: Frontiers.

37. Khaki, S., Pham, H. \& Wang, L. YieldNet: A Convolutional Neural Network for Simultaneous Corn and Soybean Yield Prediction Based on Remote Sensing Data. bioRxiv 2020.12.05.413203, DOI: 10.1101/2020.12.05.413203 (2021). Publisher: Cold Spring Harbor Laboratory Section: New Results.

38. Khaki, S., Khalilzadeh, Z. \& Wang, L. Predicting yield performance of parents in plant breeding: A neural collaborative filtering approach. PLOS ONE 15, e0233382, DOI: 10.1371/journal.pone.0233382 (2020). Publisher: Public Library of Science.

39. Merdun, H., Çınar, , Meral, R. \& Apan, M. Comparison of artificial neural network and regression pedotransfer functions for prediction of soil water retention and saturated hydraulic conductivity. Soil \& Tillage Res. 1-2, 108-116, DOI: 10.1016/j.still.2005.08.011 (2006).

40. Lamorski, K., Pachepsky, Y., Sławiński, C. \& Walczak, R. T. Using Support Vector Machines to Develop Pedotransfer Functions for Water Retention of Soils in Poland. Soil Sci. Soc. Am. J. 72, 1243-1247, DOI: 10.2136/sssaj2007.0280N (2008). _eprint: https://acsess.onlinelibrary.wiley.com/doi/pdf/10.2136/sssaj2007.0280N.

41. Landeras, G., Ortiz-Barredo, A. \& López, J. J. Comparison of artificial neural network models and empirical and semiempirical equations for daily reference evapotranspiration estimation in the Basque Country (Northern Spain). Agric. Water Manag. 95, 553-565, DOI: 10.1016/j.agwat.2007.12.011 (2008).

42. Yamaç, S. S. \& Todorovic, M. Estimation of daily potato crop evapotranspiration using three different machine learning algorithms and four scenarios of available meteorological data. Agric. Water Manag. 228, 105875, DOI: 10.1016/j.agwat. 2019.105875 (2020).

43. Obsie, E. Y., Qu, H. \& Drummond, F. Wild blueberry yield prediction using a combination of computer simulation and machine learning algorithms. Comput. Electron. Agric. 178, 105778, DOI: 10.1016/j.compag.2020.105778 (2020).

44. Rajabalizadeh, A. et al. In-depth Evaluation of APACHE Scoring System Using eICU Database. ICIS 2020 Proc. (2020).

45. Shapley, L. S. A Value for n-Person Games (Princeton University Press, 1953). Pages: 307-318 Publication Title: Contributions to the Theory of Games (AM-28), Volume II Section: Contributions to the Theory of Games (AM-28), Volume II.

46. Lundberg, S. M., Erion, G. G. \& Lee, S.-I. Consistent Individualized Feature Attribution for Tree Ensembles. arXiv preprint arXiv:1802.03888 (2019). ArXiv: 1802.03888.

47. Sundararajan, M. \& Najmi, A. The many Shapley values for model explanation. arXiv preprint arXiv:1908.08474 (2020). ArXiv: 1908.08474.

48. Vogel, E. et al. The effects of climate extremes on global agricultural yields. Environ. Res. Lett. 14, 054010, DOI: 10.1088/1748-9326/ab154b (2019). Publisher: IOP Publishing.

49. Tao, F., Xiao, D., Zhang, S., Zhang, Z. \& Rötter, R. P. Wheat yield benefited from increases in minimum temperature in the Huang-Huai-Hai Plain of China in the past three decades. Agric. For. Meteorol. 239, 1-14, DOI: 10.1016/j.agrformet. 2017.02.033 (2017).

50. Zheng, C. et al. Nighttime warming increases winter-sown wheat yield across major Chinese cropping regions. Field Crop. Res. 214, 202-210, DOI: 10.1016/j.fcr.2017.09.014 (2017).

51. Gibson, L. R. \& Paulsen, G. M. Yield Components of Wheat Grown under High Temperature Stress during Reproductive Growth. Crop. Sci. 39, 1841-1846, DOI: 10.2135/cropsci1999.3961841x (1999). _eprint: https://acsess.onlinelibrary.wiley.com/doi/pdf/10.2135/cropsci1999.3961841x. 
52. Lobell, D. B., Sibley, A. \& Ivan Ortiz-Monasterio, J. Extreme heat effects on wheat senescence in India. Nat. Clim. Chang. 2, 186-189, DOI: 10.1038/nclimate1356 (2012). Bandiera_abtest: a Cg_type: Nature Research Journals Number: 3 Primary_atype: Research Publisher: Nature Publishing Group Subject_term: Climate-change adaptation;Plant sciences Subject_term_id: climate-change-adaptation;plant-sciences.

53. Tashiro, T. \& Wardlaw, I. A Comparison of the Effect of High Temperature on Grain Development in Wheat and Rice. Annals Bot. 64, 59-65, DOI: https://doi.org/10.1093/oxfordjournals.aob.a087808 (1989). Publisher: Oxford University Press.

54. Wollenweber, B., Porter, J. R. \& Schellberg, J. Lack of Interaction between Extreme High-Temperature Events at Vegetative and Reproductive Growth Stages in Wheat. J. Agron. Crop. Sci. 189, 142-150, DOI: 10.1046/j.1439-037X.2003.00025.x (2003)._eprint: https://onlinelibrary.wiley.com/doi/pdf/10.1046/j.1439-037X.2003.00025.x.

55. Mäkinen, H. et al. Sensitivity of European wheat to extreme weather. Field Crop. Res. 222, 209-217, DOI: 10.1016/j.fcr. 2017.11.008 (2018).

56. Farooq, M., Bramley, H., Palta, J. A. \& Siddique, K. H. Heat Stress in Wheat during Reproductive and Grain-Filling Phases. Critical Rev. Plant Sci. 30, 491-507, DOI: 10.1080/07352689.2011.615687 (2011). Publisher: Taylor \& Francis _eprint: https://doi.org/10.1080/07352689.2011.615687.

57. Peichl, M., Thober, S., Meyer, V. \& Samaniego, L. The effect of soil moisture anomalies on maize yield in Germany. Nat. Hazards Earth Syst. Sci. 18, 889-906, DOI: 10.5194/nhess-18-889-2018 (2018). Publisher: Copernicus GmbH.

58. Rezaei, E. E. et al. Quantifying the response of wheat yields to heat stress: The role of the experimental setup. Field Crop. Res. 217, 93-103, DOI: 10.1016/j.fcr.2017.12.015 (2018).

59. Cannell, R. Q., Belford, R. K., Gales, K., Dennis, C. W. \& Prew, R. D. Effects of waterlogging at different stages of development on the growth and yield of winter wheat. J. Sci. Food Agric. 31, 117-132, DOI: 10.1002/jsfa.2740310203 (1980). _eprint: https://onlinelibrary.wiley.com/doi/pdf/10.1002/jsfa.2740310203.

60. Gömann, H. Wetterextreme: mögliche Folgen für die Landwirtschaft in Deutschland (2018).

\section{Acknowledgements}

The presented study has been funded by the German Federal Ministry of Education and Research (BMBF) in the framework of the funding measure 'Soil as a Sustainable Resource for the Bioeconomy - BonaRes', project BonaRes (Module A): BonaRes Center for Soil Research, subproject 'Sustainable Subsoil Management - Soil3' (grant 031B0151A).

\section{Author contributions statement}

AS and SK conceptualised the research. NS and SK contributed in model development and data analysis. AS, SK, and NS wrote the manuscript. All authors contributed to the article and approved the submitted version. 